\section{Application of the compress sensing theory for improvement of the TOF resolution in a novel J-PET instrument}

Lech Raczyński, Paweł Moskal, Paweł Kowalski, Wojciech Wiślicki, Tomasz Bednarski, Piotr Bialas, Eryk Czerwiński, Aleksander Gajos, Łukasz Kapłon, Andrzej Kochanowski, Grzegorz Korcyl, Jakub Kowal, Tomasz Kozik, Wojciech Krzemień, Ewelina Kubicz, Szymon Niedźwiecki, Marek Pałka, Zbigniew Rudy, Piotr Salabura,

Neha Gupta-Sharma, Michat Silarski, Artur Slomski, Jerzy Smyrski, Adam Strzelecki, Anna Wieczorek, Marcin Zieliński, Natalia Zoń

\begin{abstract}
Nowadays, in positron emission tomography (PET) systems, a time of flight (TOF) information is used to improve the image reconstruction process. In TOF-PET, fast detectors are able to measure the difference in the arrival time of the two gamma rays, with the precision enabling to shorten significantly a range along the line-of-response (LOR) where the annihilation occurred. In the new concept, called J-PET scanner, gamma rays are detected in plastic scintillators. In a single strip of J-PET system, time values are obtained by probing signals in the amplitude domain. Owing to compressive sensing (CS) theory, information about the shape and amplitude of the signals is recovered. In this paper, we demonstrate that based on the acquired signals parameters, a better signal normalization may be provided in order to improve the TOF resolution. The procedure was tested using large sample of data registered by a dedicated detection setup enabling sampling of signals with 50-ps intervals. Experimental setup provided irradiation of a chosen position in the plastic scintillator strip with annihilation gamma quanta.
\end{abstract}

Key words: compressed sensing • positron emission tomography • time of flight

L. Raczyński ${ }^{\bowtie}$, P. Kowalski, W. Wiślicki

Świerk Computing Centre,

National Centre for Nuclear Research,

7 Andrzeja Sołtana Str., 05-400 Otwock/Świerk, Poland,

E-mail: lech.raczynski@ncbj.gov.pl

P. Moskal, T. Bednarski, P. Białas, E. Czerwiński,

A. Gajos, G. Korcyl, J. Kowal, T. Kozik, W. Krzemień,

E. Kubicz, Sz. Niedźwiecki, M. Pałka, Z. Rudy,

P. Salabura, N. Gupta-Sharma, M. Silarski, A. Słomski,

J. Smyrski, A. Strzelecki, M. Zielinski, N. Zoń

Faculty of Physics, Astronomy and Applied Computer

Science, Jagiellonian University,

11 S. Łojasiewicza Str., 30-348 Krakow, Poland

Ł. Kapłon, A. Wieczorek

Faculty of Physics, Astronomy and Applied Computer

Science, Jagiellonian University,

11 S. Łojasiewicza Str., 30-348 Krakow, Poland and Institute of Metallurgy and Materials Science of the Polish Academy of Sciences,

25 W. Reymonta Str., 30-059 Krakow, Poland

A. Kochanowski,

Faculty of Chemistry,

Jagiellonian University,

3 R. Ingardena Str., 30-059 Krakow, Poland

Received: 28 July 2014

Accepted: 17 August 2015

\section{Introduction}

Positron emission tomography (PET) [1] is currently one of the most perspective techniques in the field of medical imaging. PET is based on the fact that the electron and positron annihilate and their mass is converted to energy in the form of two gamma quanta flying in the opposite directions. The two gamma quanta registered in coincidence define a line referred to as line of response (LOR). The image of distribution of the radionuclide is obtained from the high statistics sample of reconstructed LORs. In time of flight (TOF) PET systems [2, 3], the applied detectors measure the difference in the arrival time of the two gamma rays that enables to shorten significantly a range along the LOR used for the reconstruction of the image.

Currently, all commercial PET devices use inorganic scintillator materials, usually lutetium oxyorthosilicate (LSO) or lutetium-yttrium oxyorthosilicate (LYSO) crystals, as radiation detectors. These are characterized by relatively long rise and decay times of the order of tens of nanoseconds. The J-PET collaboration investigates a possibility of construction of a PET scanner from plastic scintillators, which would allow for simultaneous imaging of the whole human body. The J-PET chamber is built out from long strips forming the cylinder $[4,5]$. Light signals from each strip are converted into electrical 
signals by two photomultipliers (PM) placed at opposite edges. It should be noted that the better the time resolutions of the detection system, the better is the quality of a reconstructed images.

In this paper, we investigate the TOF resolution of a novel J-PET scanner and show a simple method to improve the results by applying the compressive sensing (CS) theory. In the following, we define the time resolution and present shortly the method of signal normalization based on CS theory. Then we describe an experimental setup used for signal registration and present results of improving TOF resolution in 30-cm-long plastic scintillator strip, read-out on both sides by the Hamamatsu R4998 PMs. Signals from the PMs were sampled in 50-ps steps using the Lecroy Signal Data Analyzer 6000A.

\section{Materials and methods}

\section{Definition of time resolution}

In the following, we are interested in the determination of moment of interaction of gamma quantum with a strip. The interaction moment $\left(t_{\text {hit }}\right)$ is given by

$$
t_{\text {hit }}=\frac{t_{1}+t_{2}}{2}-\frac{D}{2 v_{\text {eff }}}
$$

where $t_{1}$ and $t_{2}$ are the arrival times to the PM1 and PM2, respectively; $D$ is the length of whole strip; and $v_{\text {eff }}$ is the effective speed of the light in used scintillator. In the recent work [6], the speed of the light in the scintillator was estimated to be $12.6 \mathrm{~cm} / \mathrm{ns}$.

In order to determine the resolution (standard deviation) of $t_{\text {hit }}$ determination, an indirect method based on the estimation of the resolution of time difference $(\Delta t)$ will be provided. We assume for the sake of simplicity that $v_{\text {eff }}$ in Eq. (1) is known exactly. As the time difference $\Delta t=t_{2}-t_{1}$, we have

$$
\sigma^{2}(\Delta t)=\sigma^{2}\left(t^{1}\right)+\sigma^{2}\left(t^{2}\right)
$$

and the resolution of $t_{\text {hit }}$ based on Eq. (1) may be expressed as

$$
\sigma^{2}\left(t_{\text {hit }}\right)=\frac{\left(\sigma^{2}\left(t_{1}\right)+\sigma^{2}\left(t_{2}\right)\right)}{4}=\frac{\sigma^{2}(\Delta t)}{4}
$$

which implies that the resolution of the determination of interaction moment $\left(t_{\mathrm{hit}}\right)$ is twice better than the resolution of the time difference $(\Delta t)$.

\section{Experimental setup}

A necessary data to carry out the research has been acquired by a single module of the J-PET detector [6]. The scheme of experimental setup is presented in Fig. 1. The 30-cm-long strip was connected on two sides to the PMs. The radioactive ${ }^{22} \mathrm{Na}$ source was moved from the first to the second end in steps of $6 \mathrm{~mm}$. At each position, about 10000 pairs of signals from PM1 and PM2 were registered in coincidence with reference detector. The signals were sampled using the scope with a probing interval of 50 ps.

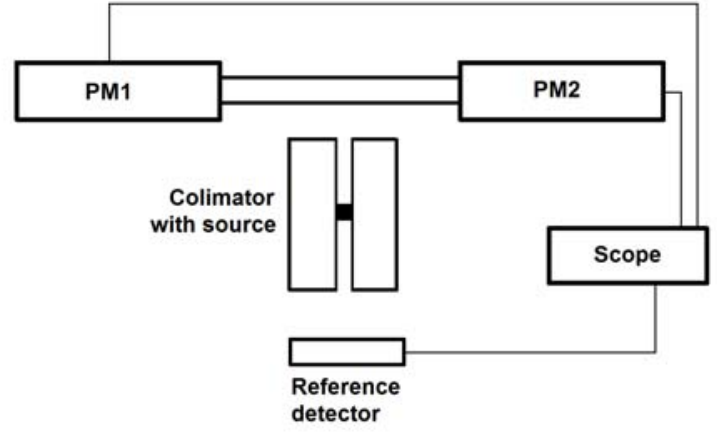

Fig. 1. Experimental setup.

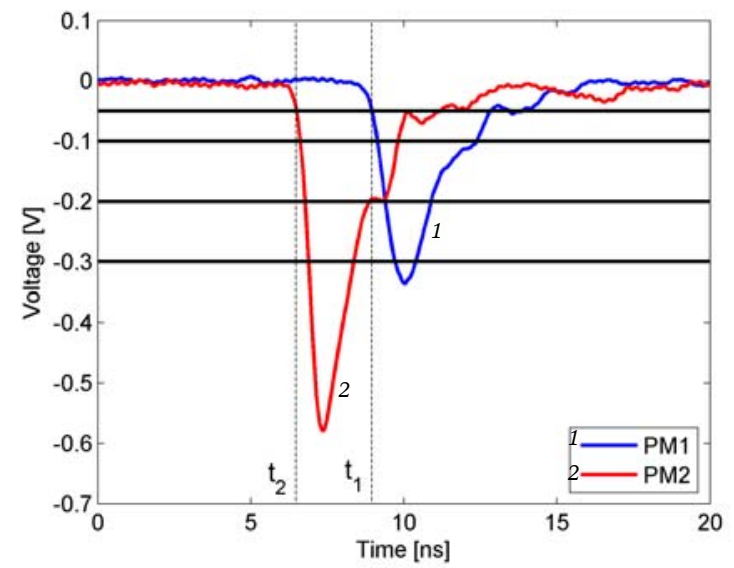

Fig. 2. Example of two signals registered in coincidence at both scintillator ends.

Examples of two signals registered at PM1 and PM2 are shown in Fig. 2 with blue and red colours, respectively, for the case when the scintillator was irradiated at distance of $7 \mathrm{~cm}$ from PM2 (23 cm from PM1).

In the final, multi-modular devices with hundreds of PMs probing with scopes will not be possible. Therefore, a multi-threshold sampling method to generate samples of a PET event waveform with respect to four user-defined amplitudes was proposed. An electronic system for probing these signals in a voltage domain was developed and successfully tested [7]. Based on the signals registered via scope, we simulate a four-level measurement with sampling in the voltage domain at 50,100, 200 and $300 \mathrm{mV}$, indicated by four black horizontal lines in Fig. 2. It should be stressed that because of the time walk effect, the resolution determined when applying the lowest threshold $(50 \mathrm{mV})$ is better with respect to the resolution obtained at the highest level $(300 \mathrm{mV})$. Therefore, the simplest way to define the start of each pulse, times $t_{1}$ and $t_{2}$, is to use the information from registration time at the lowest amplitude level, marked with vertical dashed lines in Fig. 2. From this, one may easily estimate the resolution of $\Delta t$ and, therefore, the resolution of $t_{\text {hit }}$ (see Eq. (3)).

\section{Compressive sensing framework}

As the shape and amplitude of signals are predominantly related with the hit position, further improvement of the resolution may be provided by 
the analysis of the full-time signals. According to the theory of CS [8,9], a signal that is sparse in some domain can be recovered based on far fewer samples than required by the Nyquist sampling theorem. In the recent articles [10-12], we have proposed a novel signal recovery scheme based on the CS method and the statistical analysis that fits to the signal processing scenario in J-PET devices. Under this theory, only a recovery of sparse or compressible signals is possible. In articles $[10,11]$, the sparse representation of signals was provided by the principal component analysis (PCA) decomposition. We will not describe all the steps of signal processing here, but we just state that the recovery of full-time signals based on eight samples is very accurate. For further details about signal recovery scheme in the J-PET framework, the interested reader is referred to Refs. [10-12].

The application of CS theory enables to take an advantage from fully sampled signals and opens an area for completely new algorithms for the estimation of the values of times $t_{1}$ and $t_{2}$ (see Fig. 2). In the following, we use the recovered signals to provide the signal normalization.

\section{Method of signal normalization}

Owing to the low detection efficiency of the plastic scintillators (and interaction of gamma quanta predominantly via Compton effect), low number of photons reach the PMs and the charge, as well as amplitude, of signals is subject to a large variations. However, the shapes of the signals are highly related with the position of the interaction. In order to improve the time resolution, a method of signal normalization that permits to decrease the smearing of signals charge is proposed. The procedure of signal normalization is as follows.

Consider $L$ data sets representing charges of signals at PM1 and PM2 gathered for $L$ positions. The mean values of the charges at positions along the strip $(i=1, \ldots, L)$ at PM1 and PM2 will be denoted by $Q_{m 1}(i)$ and $Q_{m 2}(i)$, respectively. Furthermore, the standard deviation of charges at each position along the strip $(i=1, \ldots, L)$ at PM1 and PM2 will be denoted by $Q_{s 1}(i)$ and $Q_{s 2}(i)$, respectively.

Suppose that new pair of signals $S_{1}$ and $s_{2}$ have been recovered based on time samples registered at four amplitudes levels at PM1 and PM2, respectively. The charges of the signals, $Q_{1}$ and $Q_{2}$, are calculated as an integrations of the $s_{1}$ and $s_{2}$ functions, respectively. The proposed normalization procedure qualifies a new measurement, represented by $Q_{1}$ and $Q_{2}$, to the $j$-th position:

$$
j=\arg \min \left(\left(Q_{m 1}(i)-Q_{1}\right)^{2}+\left(Q_{m 2}(i)-Q_{2}\right)^{2}\right)
$$

Next, the recovered signals $s_{1}$ and $s_{2}$ are normalized according to the formula:

$$
\begin{aligned}
& s_{n 1}=s_{1} \cdot Q_{m 1}(j) / Q_{1} \\
& s_{n 2}=s_{2} \cdot Q_{m 2}(j) / Q_{2}
\end{aligned}
$$

where $s_{n 1}$ and $s_{n 2}$ denote the normalized signals.

\section{Experimental results}

In the first step of the analysis, the charge distributions of signals were investigated. Experimental results based on the signals registered along the scintillator strip are presented in Fig. 3. Mean values of charges at PM1 and PM2 are marked with solid blue and red curves, respectively. As expected, the curves are symmetrical with respect to the centre of the scintillator strip (position of $15 \mathrm{~cm}$ ). The distributions of $Q_{m 1} \pm Q_{s 1}$ and $Q_{m 2} \pm Q_{s 2}$ at PM1 and PM2 along the strip are marked with dashed blue and red curves, respectively. From Fig. 3, one may observe that $Q_{s 1}$ and $Q_{s 2}$ have the same trend as $Q_{m 1}$ and $Q_{m 2}$, respectively, and are in the range from about 6 to $20 \mathrm{pC}$.

\section{An example of the signal normalization}

Figure 4 shows an example of the normalization of signals registered at PM1 at fixed position $5 \mathrm{~cm}$ from PM1. The three corresponding signals registered at second PM (PM2) are not shown here but were used during the normalization process to estimate the position of irradiation (see Eq. (4)). Figure 4a shows a three randomly selected raw signals registered via scope. Figure $4 \mathrm{~b}$ presents the same signals after the normalization procedure provided according to the description given in section 'Method of signal normalization'. The same colors of the signals in Fig. 4a and $4 \mathrm{~b}$ indicate a corresponding pair of signals before and after normalization. As it is seen from Fig. 4a, the shapes of the three signals are similar but the charges differ. However, an estimated positions according to Eq. (4) were found to be very close, and therefore, the charges of the normalized signals were also very similar (see Fig. 4a).

\section{Time resolution of the event time reconstruction}

The normalization method was verified using signals from all the irradiation positions. Each pair of signals was recovered via CS method based on the information from four amplitude levels (see Fig. 2) and then normalized according to the description given in section 'Method of signal normalization'. In order to compare the time resolutions before and after normalization process, the normalized signals, $s_{n 1}$ and $s_{n 2}$, were sampled in the voltage domain at four levels from 50 to $300 \mathrm{mV}$ (see Fig. 2). For each position and at each level, the distribution of time difference was calculated.

In Figs. 5 and 6 , the resulting resolutions $(\sigma(\Delta t))$ are presented as a function of irradiated position, determined when applying the lowest threshold $(50 \mathrm{mV})$ and the highest threshold $(300 \mathrm{mV})$, respectively. In Figs. 5 and 6, the resolutions obtained based on the raw and normalized signals are indicated with blue and red squares, respectively. As expected, because of the time walk effect, the resolution determined when applying threshold at $50 \mathrm{mV}$ is better with respect to the resolution ob- 


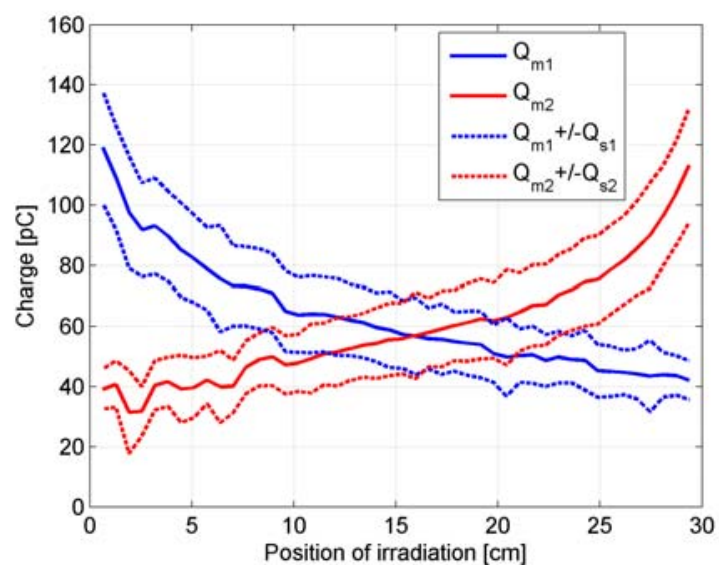

Fig. 3. Charge distributions along the scintillator strip.
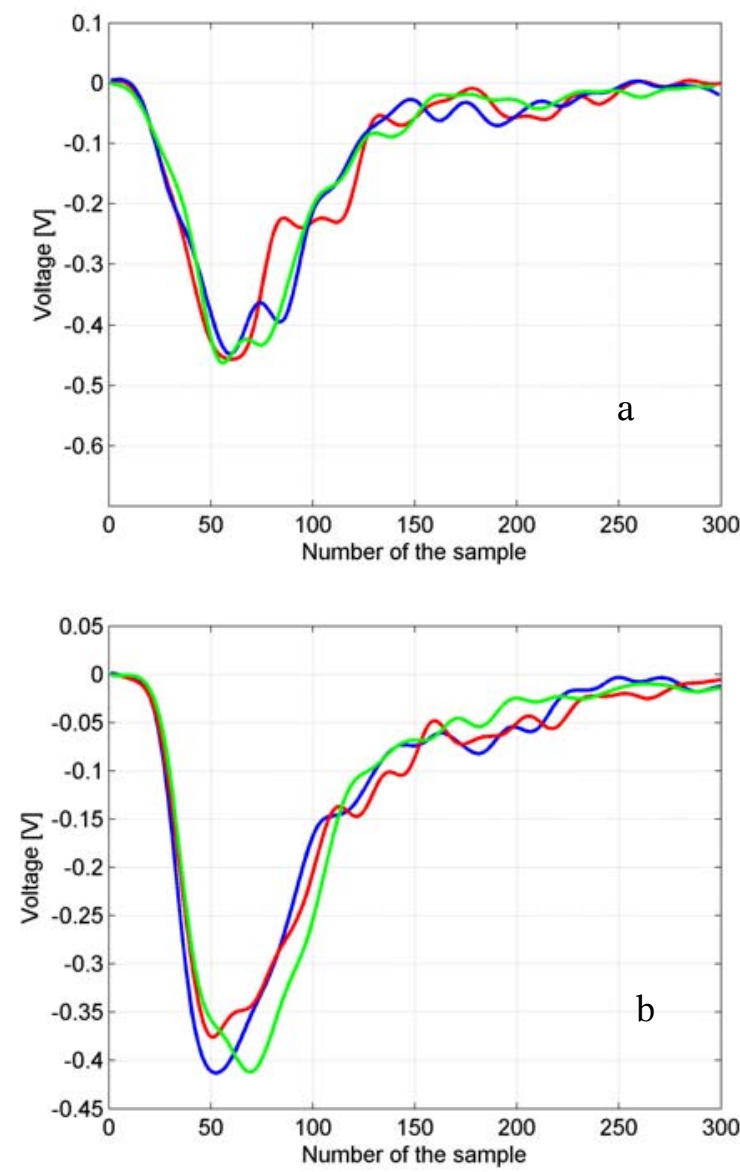

Fig. 4. Example of signals before (a) and after (b) the normalization process.

tained at $300 \mathrm{mV}$. However, the influence of time walk effect is more visible in the case of raw signals. From Figs. 5 and 6, one can infer that the time resolution is almost independent of the position of irradiation. An average resolution of the time difference along the strip at the lowest tested amplitude level (Fig. 5) was determined to be 172 and 160 ps for the raw and normalized signals, respectively. This corresponds to the improvement of the resolution of the moment of the interaction from 86 ps to about 80 ps.

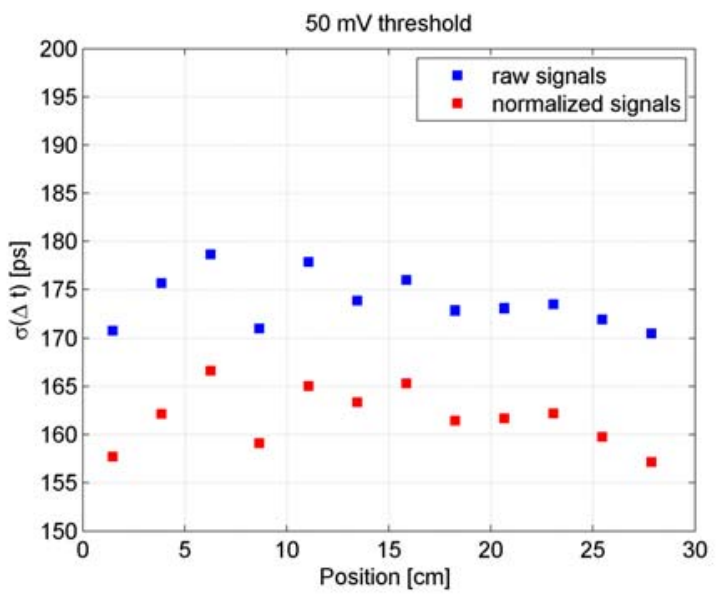

Fig. 5. Time resolution of the reconstruction after appying the lowest amplitude thresold of $50 \mathrm{mV}$.

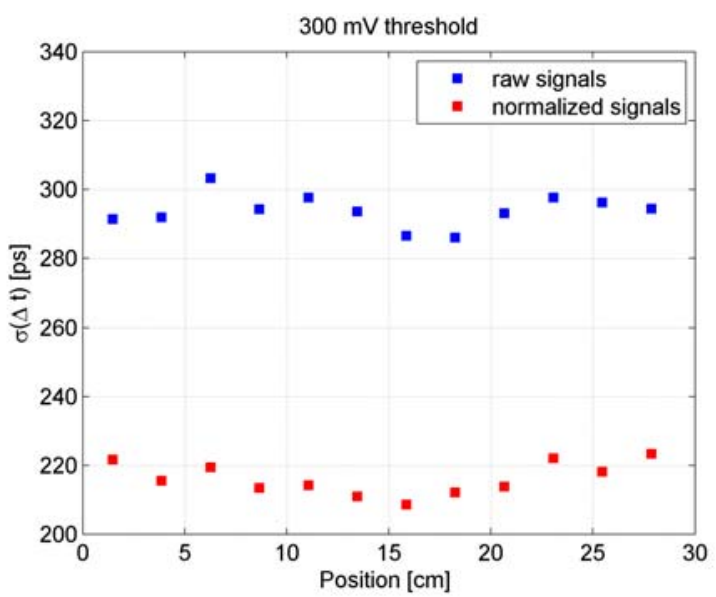

Fig. 6. Time resolution of the reconstruction after appying the highest amplitude thresold of $300 \mathrm{mV}$.

\section{Conclusions}

In this paper, the concept of signal normalization in a novel J-PET scanner was introduced. J-PET device is based on plastic scintillators and, therefore, is a promising solution in view of the TOF resolution. In the related works $[10,11]$, it was shown that CS theory can be successfully applied to the problem of signal recovery in a J-PET scanner. The information from fully recovered signals was used in order to provide the normalization of the signals. It was shown that with fully recovered signals, a better time resolution of J-PET scanner is achieved; the resolution of the moment of the interaction was improved from about 86 to $80 \mathrm{ps}$. It should be stressed that different approaches for using the recovered information from CS theory may be considered and the studies are in progress.

Acknowledgments. We acknowledge technical and administrative support by T. Gucwa-Rys, A. Heczko, M. Kajetanowicz, G. Konopka-Cupiał and W. Migdał and the financial support by the Polish National Center for Development and Research through grant no. INNOTECH-K1/IN1/64/159174/NCBR/12, the Foun- 
dation for Polish Science through MPD programme and the EU, MSHE grant no. POIG.02.03.00-161 00-013/09 and Doctus - the Malopolska PhD Scholarship Fund.

\section{References}

1. Humm, J. L., Rosenfeld, A., \& Del Guerra, A. (2003). From PET detectors to PET scanners. Eur. J. Nucl. Med. Mol. Imaging, 30(11), 1574-1597.

2. Karp, J. S., Surti, S., Daube-Witherspoon, M. E., \& Muehllehner, G. (2008). Benefit of time-of-flight in PET: Experimental and clinical results. J. Nucl. Med., 49(3), 462-470.

3. Conti, M. (2009). State of the art and challenges of time-of-flight PET. Phys. Med., 25, 1-11.

4. Moskal, P., Salabura, P., Silarski, M., Smyrski, J., Zdebik, J., \& Zieliński, M. (2011). Novel detector systems for the Positron Emission Tomography. Bio-Algorithms and Med-Systems, 7, 73-78. [arXiv:1305.5187].

5. Moskal, P., Bednarski, T., Białas, P., Czerwiński, E., Kapłon, Ł., Kochanowski, A., Korcyl, G., Kowal, J., Kowalski, P., Kozik, T., Krzemień, W., Molenda, M., Niedźwiecki, Sz., Pałka, M., Pawlik, M., Raczyński, L., Rudy, Z., Salabura, P., Gupta-Sharma, N., Silarski, M., Słomski, A., Smyrski, J., Strzelecki, A., Wiślicki, W., Zieliński, M., \& Zoń, N. (2014). A novel TOF-PET detector based on organic scintillators. Radiother. Oncol., 110, S69-S70.

6. Moskal, P., Niedźwiecki, Sz., Bednarski, T., Czerwiński, E., Kapłon, Ł., Kubicz, E., Moskal, I., Pawlik-Niedźwiecka, M., Sharma, N. G., Silarski, M., Zieliński, M., Zoń, N., Białas, P., Gajos, A., Kochanowski, A., Korcyl, G., Kowal, J., Kowalski, P., Kozik, T., Krzemień, W., Molenda, M., Pałka, M., Raczyński, L., Rudy, Z., Salabura, P., Słomski, A., Smyrski, J., Strzelecki, A., Wieczorek, A., \& Wiślicki, W. (2014). Test of a single module of the J-PET scanner based on plastic scintillators. Nucl. Instrum. Methods Phys. Res. Sect. A-Accel. Spectrom. Dect. Assoc. Equip., 764, 317-321. DOI: 10.1016/j.nima.2014.07.052. [arXiv:1407.7395].

7. Pałka, M., Moskal, P., Bednarski, T., Białas, P., Czerwiński, E., Kapłon, Ł., Kochanowski, A., Korcyl, G., Kowal, J., Kowalski, P., Kozik, T., Krzemień,
W., Molenda, M., Niedźwiecki, Sz., Pawlik, M., Raczyński, L., Rudy, Z., Salabura, P., Gupta-Sharma, N., Silarski, M., Słomski, A., Smyrski, J., Strzelecki, A., Wiślicki, W., Zieliński, M., \& Zoń, N. (2014). A novel method based solely on FPGA units enabling measurement of time and charge of analog signals in Positron Emission Tomography. Bio-Algorithms \& Med-Systems, 10, 41-45.

8. Candès, E., Romberg, J., \& Tao, T. (2006). Robust uncertainty principles: Exact signal reconstruction from highly incomplete frequency information. IEEE Trans. Inform. Theory, 52, 489-509.

9. Donoho, D. (2006). Compressed sensing. IEEE Trans. Inform. Theory, 52, 1289-1306.

10. Raczyński, L., Kowalski, P., Bednarski, T., Białas, P., Czerwiński, E., Kapłon, Ł., Kochanowski, A., Korcyl, G., Kowal, J., Kozik, T., Krzemień, W., Molenda, M., Moskal, P., Niedźwiecki, Sz., Pałka, M., Pawlik, M., Rudy, Z., Salabura, P., Gupta-Sharma, N., Silarski, M., Słomski, A., Smyrski, J., Strzelecki, A., Wiślicki, W., \& Zieliński, M. (2013). Application of compressive sensing theory for the reconstruction of signals in plastic scintillators. Acta Phys. Pol. B-Proc. Suppl., 6(4), 1121-1127.

11. Raczyński, L., Moskal, P., Kowalski, P., Wiślicki, W., Bednarski, T., Białas, P., Czerwiński, E., Gajos, A., Kapłon, Ł., Kochanowski, A., Korcyl, G., Kowal, J., Kozik, T., Krzemień, W., Kubicz, E., Niedźwiecki, Sz., Pałka, M., Rudy, Z., Rundel, O., Salabura, P., Gupta-Sharma, N., Silarski, M., Słomski, A., Smyrski, J., Strzelecki, A., Wieczorek, A., Zieliński, M., \& Zoń, N. (2015). Compressive sensing of signals generated in plastic scintillators in a novel J-PET instrument. Nucl. Instrum. Methods Phys. Res. Sect. A-Accel. Spectrom. Dect. Assoc. Equip., 786, 105-112.

12. Raczyński, L., Moskal, P., Kowalski, P., Wiślicki, W., Bednarski, T., Białas, P., Czerwiński, E., Kapłon, Ł., Kochanowski, A., Korcyl, G., Kowal, J., Kozik, T., Krzemień, W., Kubicz, E., Molenda, M., Moskal, I., Niedźwiecki, Sz., Pałka, M., Pawlik- Niedźwiecka, M., Rudy, Z., Salabura, P., Gupta-Sharma, N., Silarski, M., Słomski, A., Smyrski, J., Strzelecki, A., Wieczorek, A., Zieliński, M., \& Zoń, N. (2014). Novel method for hit-position reconstruction using voltage signals in plastic scintillators and its application to Positron Emission Tomography. Nucl. Instrum. Methods Phys. Res. Sect. A-Accel. Spectrom. Dect. Assoc. Equip., 764, 186-192. 